\section{Medicina Baseada em Euidências \\ TOXINA BOTULÍNICA OU ESFINCTEROTOMIA LATERAL PARA O TRATAMENTO DA FISSURA ANAL CRÔNICA?}

O objetivo do tratamento para fissuras anais é induzir uma redução temporária da pressão do canal anal para promover a sua cicatrização permanentemente, sem perturbar a função normal do esfíncter anal. Uma redução no tônus do esfíncter anal é alcançado através do relaxamento do esfíncter anal interno, sob ação direta de suas células musculares lisas. Essas alterações podem ocorrer com auxílio de fármacos como a toxina botulínica.

A toxina botulínica é uma neurotoxina, com potente ação na sinapse neuromuscular, onde atua inibindo a liberação de acetilcolina pelo axônio do neurônio motor e diminuindo a reatividade da fibra muscular lisa a esse neurotransmissor, bloqueando a contração muscular. Assim, há tempo suficiente para a cicatrização da fissura.

Para se obter a melhor evidência na resposta à questão, realizou-se busca na base dados MEDLINE, a fim de recuperar ensaios clínicos controlados e randomizados comparando o tratamento químico ao cirúrgico. Foram utilizadas as seguintes palavras-chave: "Fissure in ano", "Botulinum toxins" e "Randomized Controlled Trial". Apenas estudos publicados em inglês, espanhol ou português, e com pontuação igual ou maior a três na escala Jadad ${ }^{1}$, foram selecionados para análise. Os desfechos analisados foram cura e recorrência da fissura.

Os resultados foram sustentados nos dados de quatro estudos ${ }^{2-5}$. A síntese permitiu obter as seguintes conclusões referentes à cura e a recorrência nos respectivos períodos de seguimento:

\section{CURA}

- Quatro semanas (Um mês): Aumento do risco absoluto (ARA) com toxina botulínica do paciente não ser curado variando entre $19,7 \%$ e $36 \%$ (IC95\% 3,5\% a 54,8\%), com $\mathrm{NNH}$ variando de 3 a 5 (IC95\% 2 a 28), quando comparado à esfincterotomia lateral;

- Seis semanas: 0 tratamento com toxina botulínica aumenta o risco absoluto do paciente não ser curado em 44,5\% (IC95\% $16,7 \%$ a $72,3 \%$ ), com NNH igual a 2 (IC95\% 1 a 6) quando comparado à esfincterotomia lateral;

- Oito semanas (Dois meses): O tratamento com toxina botulínica aumenta o risco absoluto do paciente não obter cura variando entre $24,2 \%$ a $36 \%$ (IC95\% 12,5\% a 54,8\%), com $\mathrm{NNH}$ variando entre 3 e 4 (IC95\% 2 a 8), quando comparado à esfincterotomia lateral;

- Vinte e seis semanas (Seis meses): O tratamento com toxina botulínica aumenta o risco absoluto do paciente não ser curado em 49,3\% (IC95\% 22,8\% a 75,8\%), com NNH igual a 2 (IC95\% 1 a 4) quando comparado à esfincterotomia lateral;

- Doze meses: O tratamento com toxina botulínica aumenta o risco absoluto do paciente não obter cura variando entre $18,6 \%$ a $47,5 \%$ (IC95\% 5,9\% a 64,9\%), com NNH variando entre 2 e 5 (IC95\% 2 a 17), quando comparado à esfincterotomia lateral;

\section{RECORRÊNCIA}

- O tratamento com toxina botulínica aumenta o risco absoluto de o paciente apresentar recorrência em 13,2\% (IC95\% $4,1 \%$ a $22,3 \%$ ), com NNH igual a 8 (IC95\% 4 a 24) em um seguimento médio de 12 meses.

0 resultado da combinação dos ensaios indica a inexistência de benefício do tratamento farmacológico com toxina botulínica das fissuras anais crônicas comparada ao tratamento cirúrgico.

\section{Felipe Toyama Aires ${ }^{1}$ Romulo Paris Soares ${ }^{1}$ Wanderley Marques Bernardo ${ }^{2}$ Sérgio Eduardo Alonso Araujo ${ }^{3}$}

1 - Acadêmico da Faculdade de Medicina - UNILUS, Santos, SP 2 - Doutor pela Universidade de São Paulo na área de Cirurgia Torácica, Especialização em Medicina Baseada em Evidências EBM Centre - Oxford e Especialização no Desenvolvimento de Ensaios Randomizados pela Universidade de Oxford. Membro do Comitê Técnico do Programa Diretrizes AMB/CFM, São Paulo, SP 3 - Doutor pela Faculdade de Medicina da Universidade de São Paulo na área de Cirurgia do Aparelho Digestivo do Departamento de Gastroenterologia, Especialista em Cirurgia do Aparelho Digestivo e em Coloproctologia, São Paulo, SP

\section{Referências}

1. Jadad AR, Moore RA, Carroll D, Jenkinson C, Reynolds DJ, Gavaghan DJ, McQuay HJ. Assessing the quality of reports of randomized clinical trials: is blinding necessary? Control Clin Trials, 1996;17:1-12.

2. Menteş BB, İrkörücü O, Akin M, leventoğlu S, Tatlicioğlu E. Comparison of botulinum toxin injection and lateral internal sphincterotomy for the treatment of chronic anal fissure. Dis Colon Rectum 2003;46:232-237.

3. Arroyo A, Pérez F, Serrano P, Candela F, Lacueva J, Calpena R. Surgical versus chemical (botulinum toxin) sphincterotomy for chronic anal fissure: long-term results of a prospective randomized clinical and manometric study. Am J Surg 2005; 189:429-434.

4. Iswariah H, Stephens J, Rieger N, Rodda D, Hewett P. Randomized prospective controlled trial of lateral internal sphincterotomy versus injections of botulinum toxin for the treatment of idiopathic fissure in ano. ANZ J Surg 2005; 75:553-555.

5. Massoud BW, Mehrdad V, Baharak T, Alireza Z. Botulinum toxin injection versus internal anal sphincterotomy for the treatment of chronic anal fissure. Ann Saudi Med 2005;25(2):140-142. 